\title{
PENETRATING TRAUMA IN UPPER ABDOMEN CAUSING ISOLATED POSTERIOR GASTRIC WALL PERFORATION: A RARE PRESENTATION
}

Mudit Agarwal ${ }^{1}$, Surender Kumar²

\section{HOW TO CITE THIS ARTICLE:}

Mudit Agarwal, Surender Kumar. "Penetrating Trauma in Upper Abdomen Causing Isolated Posterior Gastric Wall Perforation: A Rare Presentation". Journal of Evolution of Medical and Dental Sciences 2015; Vol. 4, Issue 33, April 23; Page: 5773-5775, DOI: 10.14260/jemds/2015/844

ABSTRACT: Isolated posterior gastric wall perforation is extremely rare injury in cases of penetrating injury to upper abdomen. We report a case of a 20 years old male who presented to casualty with alleged history of penetrating injury to upper abdomen with a knife. Radiological investigations were normal on presentation. During observation patient developed signs of peritonitis. Patient underwent laparotomy and was found to have isolated posterior gastric wall perforation. Primary repair of perforation was done. Post op. period was uneventful. Such injuries may have minimal clinical or radiologic signs early in the course of presentation leading to delayed presentation. So a high degree of suspicion is required to diagnose these conditions early to decrease morbidity and mortality.

KEYWORDS: Penetrating trauma; isolated posterior gastric wall perforation; rare presentation.

INTRODUCTION: The anterior abdominal wall is defined as the area between the xiphoid and the pubic symphysis, and both posterior axillary lines. The danger to the enteric structures lies with the potential that the stabbing instrument could have traversed peritoneum and penetrated stomach, small bowel or transverse colon. The diagnostic challenge of multiple visceral injuries, the notorious difficulty of establishing the proper sequence for intervention, the injury severity and frequent hemodynamic instability and the inherent danger of septicaemia conspire to increase morbidity and mortality for these injuries.

CASE REPORT: A 20 year old male patient presented to emergency department with alleged history of homicidal stab wound by a knife in upper abdomen. History suggested only 1 inch penetration. Patient complained of pain at the site of stab wound and had no complaints of breathing difficulty or abdominal pain. On presentation vitals were stable. Systemic examination was normal. Penetrating wound was present about $2 \mathrm{~cm}$ below the xiphisternum. No evisceration was present. On digital exploration of wound, peritoneal breach was present. Chest X-ray, X-ray Erect abdomen and Ultrasound of the abdomen were normal. Patient was admitted for observation. Patient was asymptomatic for more than 24 hours. After 36 hours of admission patient complained of increase in the severity of pain in upper abdomen. Repeat erect X-ray of the abdomen showed gas under diaphragm and gradually patient developed signs of peritonitis. Patient was posted for emergency laparotomy and was found to have perforation of stomach on its posterior wall (Fig. 1) measuring 3. $0 \mathrm{x} 0.5 \mathrm{cms}, 500 \mathrm{ml}$ of bile stained fluid was present in peritoneal cavity. No other abdominal organs were injured. Perforation site was closed primarily and abdominal drains were put. Drains were removed on postoperative day 6. Post-operative period was uneventful. 


\section{CASE REPORT}

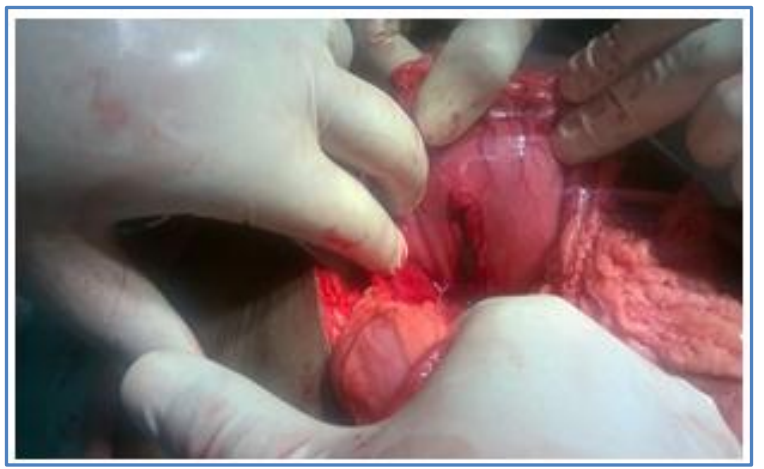

Fig. 1: Posterior wall perforation

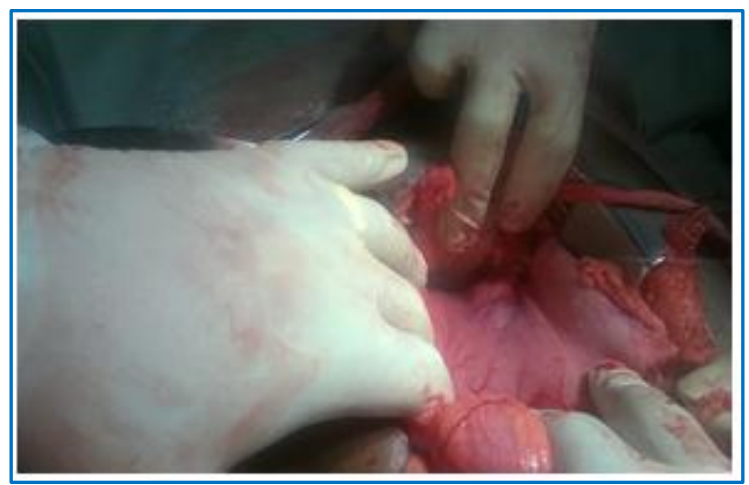

Fig. 2: Normal anterior wall

DISCUSSION: Stab wounds to the anterior abdomen have been shown to be associated with a 30 $50 \%$ incidence of intra-abdominal injury that requires operative repair.[1][2] Therefore, over half of the patients can be discharged without an operation. In a study by Moore et al the frequency of abdominal organs involved in penetrating thoracoabdominal trauma by a stab wound was liver (30\% cases), diaphragm (12\%), spleen (5\%), stomach (1\%), colon (3\%), and small bowel (2\%).[3] Injuries to stomach almost always consist of single or pair of perforations. The site most commonly affected is the anterior wall (40\%), followed by greater curvature (23\%), lesser curvature $15 \%)$ and posterior wall (15\%).[4,5,6]

Physical examination and hemodynamic monitoring are accurate tools for assessing the need for abdominal exploration. Indications for operation include abdominal tenderness away from the sight of injury, hypotension, or ongoing hemorrhage. The presence of peritoneal penetration, evisceration of omentum or bowel, abdominal paracentesis positive for blood, air under the diaphragm on x-ray, and shock are not absolute indications for surgery.[7] Those patients not requiring immediate laparotomy, require close observation. Delayed manifestations of intraabdominal injuries may take up to 24 hours to present. In the above case signs of peritonitis developed after 36 hours. Any unexplained free fluid in the abdomen on CT scan after a penetrating injury mandates abdominal exploration. Alternate diagnostic approach could be use of peritoneal lavage and diagnostic laparoscopy.

Intraoperative evaluation of stomach begins with full visualisation of anterior gastric surface from pylorus to esophagogastric junction. The posterior aspect of stomach is assessed by opening the 
gastrocolic ligament. Care must be taken to avoid injuring the vascular aracade of greater curvature. Identified stomach perforations and lacerations should have the traumatized edges resected. As the stomach is very vascular it should be closed in two layers, to ensure hemostasis: a full thickness inner layer of an absorbable running suture followed by an interrupted Lembert or seromuscular absorbable suture. If there is excessive hemorrhage then the inner running suture layer may also be locked. In the pyloric area, a pyloroplasty should be performed to avoid pyloric stenosis. If there is extensive destruction then a partial gastrectomy may be performed with a Billroth I anastomosis if there is enough duodenum or a Billroth II, gastrojejunostomy if the proximal duodenum is also damaged. Hematomas of the wall should be evacuated; hemostasis achieved and closure with absorbable Lembert sutures only if no full thickness injury is encountered. Drainage of primary repairs or anastomotic lines on the stomach is not usually necessary.

CONCLUSION: Penetrating upper abdomen injuries are associated with high risk of injury to diaphragm and enteric organs. Such injuries may have minimal clinical or radiologic signs early in the course of presentation leading to delayed presentation. So a high degree of suspicion is required to diagnose these conditions early to decrease morbidity and mortality.

\section{REFERENCES:}

1. Demetriades D, Rabinowitz B. Indications for operation in abdominal stab wounds. Ann Surg 1987 Feb. 205 (2): 129-32.

2. Shorr RM, Gottlieb MM, WebbK, Ishiguro L. Berne TV. Selective management of abdominal stab wounds. Arch Surg 1988 Sep. 123 (9): 1141-5.

3. Moore JB, Moore EE, Thompson JS; Abdominal injuries associated with penetrating trauma in the lower chest. Am J Surg, 1980; 140: 724-730.

4. Rodríguez-Hermosa JI, Roig J, Sirvent JM, Codina-Cazador A, Gironès J, Puig J, Osorio M; Gastric perforations from abdominal trauma. Dig Surg, 2008; 25 (2): 109-116.

5. Nanji SA, Mock C; Gastric rupture resulting from blunt abdominal trauma and requiring gastric resection. J Trauma, 1999; 47 (2): 410- 412.

6. Deshpande AD, Sivapragasam S; Isolated posterior gastric injury due to blunt abdominal trauma. Emerg Med J, 2003; 20 (6): 566.

7. fordan A. Weinberg; Timothy C. Fabian; injuries to the stomach, small bowel, colon and rectum, ACS Surgery2005.

\section{AUTHORS:}

1. Mudit Agarwal

2. Surender Kumar

\section{PARTICULARS OF CONTRIBUTORS:}

1. Senior Resident, Department of General Surgery, KGMU.

2. Associate Professor, Department of General Surgery, KGMU.

FINANCIAL OR OTHER

COMPETING INTERESTS: None

\section{NAME ADDRESS EMAIL ID OF THE} CORRESPONDING AUTHOR:

Dr. Mudit Agarwal, \# 67, Rajendra Nagar, Near Mangla Traders, Bijnor, Uttar Pradesh. E-mail: muditkgmu1807@yahoo.co.in

Date of Submission: 27/03/2015.

Date of Peer Review: 28/03/2015.

Date of Acceptance: 11/04/2015.

Date of Publishing: 23/04/2015. 